\title{
Asociación del consumo de fibra dietética con la actividad de la colitis ulcerosa crónica idiopática. Estudio exploratorio en población mexicana
}

Cinthya J. Meza-Ortiz, Sophia E. Martínez-Vázquez y Jesús K. Yamamoto-Furusho*

Departamento de Gastroenterología, Instituto Nacional de Ciencias Médicas y Nutrición "Salvador Zubirán”, Ciudad de México, México

\section{Resumen}

Introducción: La fibra dietética suplementada coadyuva en la remisión de la colitis ulcerosa crónica idiopática (CUCl). Objetivo: Evaluar la asociación entre la cantidad de fibra de la dieta habitual y la actividad de la enfermedad en pacientes con CUCI. Métodos: Estudio transversal de una de cohorte de pacientes con CUCl. Se calculó el consumo de fibra dietética y con $U$ de Mann-Whitney se comparó con la cantidad recomendada (14 g/1000 kcal). Con coeficiente de correlación de Spearman se analizaron el número de recaídas y la cantidad de fibra consumida, y la relación de esta con la actividad de la enfermedad mediante regresión logística. Resultados: El grupo sin actividad actual de CUCl consumió más fibra dietética $(R I C=18-26, p=0.062)$ y $47 \%$ consumió más de la cantidad recomendada; el análisis de regresión logística mostró que cumplir con esta se asoció como factor protector en contra de la actividad actual de la CUCl $(R M=0.227, p=0.032)$. En la fibra y el número de recaídas en el último año se observó una correlación inversamente proporcional $(r=-0.399, p=0.011)$. Conclusiones: El consumo recomendado de fibra en la población general tuvo un efecto protector para la actividad actual de la $\mathrm{CUCl}$ en pacientes mexicanos.

PALABRAS CLAVE: Fibra. Dieta. Colitis ulcerosa crónica idiopática.

Association of dietary fiber consumption with disease activity in ulcerative colitis. An exploratory study in the Mexican population

\begin{abstract}
Introduction: Dietary fiber intake helps in the remission of ulcerative colitis (UC). Objective: To evaluate if there is an association between the amount of fiber in usual diet and disease activity in patients with UC. Methods: Cross-sectional study of a cohort of patients with UC. Dietary fiber intake was calculated and compared with the recommended amount (14 g/1000 kcal) with Mann-Whitney's U-test. Using Spearman's correlation coefficient, the number of relapses and the amount of consumed fiber were analyzed, and the relationship of dietary fiber consumption with disease activity was established by logistic regression. Results: The group without ongoing UC activity consumed a higher amount of dietary fiber $(20 \mathrm{~g}, 1 Q R=18-26$, $p=0.062$ ), and $47 \%$ consumed more than the recommended amount; the logistic regression analysis showed that compliance with recommended fiber consumption was associated as a protective factor against UC current activity $(O R=0.227$, $p=0.032)$. As for dietary fiber intake and the number of relapses within previous year, an inversely proportional correlation was observed ( $r=-0.399, p=0.011$ ). Conclusions: Consumption of the recommended dietary fiber amount in the general population had a protective effect against UC activity in Mexican patients.
\end{abstract}

KEYWORDS: Fiber. Diet. Ulcerative colitis.

Correspondencia:

*Jesús K. Yamamoto-Furusho

E-mail: kazuofurusho@hotmail.com

0016-3813/@ 2021 Academia Nacional de Medicina de México, A.C. Publicado por Permanyer. Este es un artículo open access bajo la licencia CC BY-NC-ND (http://creativecommons.org/licenses/by-nc-nd/4.0/).
Fecha de recepción: 20-07-2021

Fecha de aceptación: 21-09-2021

Gac Med Mex. 2022;158:43-49

Disponible en PubMed
www.gacetamedicademexico.com ww.gacetamedicademexico.com

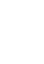




\section{Introducción}

La colitis ulcerosa crónica idiopática (CUCl) es una enfermedad inflamatoria del intestino, en la cual la interacción de factores genéticos, inmunitarios y ambientales afecta la mucosa del colon y recto, ${ }^{1,2} \mathrm{En}$ México, entre 1987 y 2006, su incidencia se incrementó de 28.8 a 76.1 casos nuevos. ${ }^{3}$ Los síntomas y la gravedad de esta enfermedad dependen de la extensión, grado de inflamación y manifestaciones extraintestinales asociadas: ${ }^{1}$ la confirmación se establece con algunos marcadores séricos, endoscópicos e histopatológicos. ${ }^{4}$ El tratamiento dependerá del grado de actividad inflamatoria y el patrón anatomoclínico de cada paciente. ${ }^{5}$ Se ha descrito que el objetivo del tratamiento es curar la mucosa; ${ }^{4}$ en ese sentido, parece que la fibra dietética suplementada puede coadyuvar en la remisión de la enfermedad. ${ }^{6}$ La fibra son hidratos de carbono que pueden ser hidrolizados y fermentados por el microbioma intestinal; en la dieta, la fibra está en cereales integrales, leguminosas, frutas y verduras. ${ }^{7}$ La recomendación actual de consumo de fibra es de $14 \mathrm{~g}$ por cada 1000 calorías. ${ }^{8}$ Los principales productos de esta fermentación son los ácidos grasos de cadena corta (AGCC): acetato, propionato y butirato; gases y energía. El butirato es utilizado por los colonocitos y metabolizado hasta dióxido de carbono, cuerpos cetónicos y agua; además, disminuye la producción de citoquinas, como el factor de necrosis tumoral (TNF). ${ }^{9}$ Aunado a esto, los AGCC producidos al metabolizar la fibra han demostrado estimular la absorción de agua, sodio en el colon y promover la curación de la mucosa, lo que ayuda a que los pacientes en remisión se mantengan en este periodo. ${ }^{10}$

Diversos estudios investigan la relación del alto consumo de fibra dietética no suplementada y la reducción del riesgo de padecer enfermedad inflamatoria intestinal o recaída de la enfermedad explicada por modificaciones en el microbioma intestinal, $, 8,11-13$ así como por disminución en la betaoxidación del butirato luminal, lo que ocasionaría un déficit energético en el epitelio, proceso relacionado con la patogénesis de la enfermedad. ${ }^{14,15}$ Estudios experimentales con fibra suplementada han sugerido que el butirato inhibe la producción de algunas citoquinas y la activación del factor de transcripción NF-kB, lo que constituye una evidencia de su efecto antiinflamatorio. ${ }^{16,17}$ Una revisión sistemática de ensayos clínicos concluyó que la $\mathrm{CUCl}$ es susceptible al tratamiento con fibra soluble, ${ }^{18}$ pero no queda claro el papel de la fibra dietética no suplementada, situación que pudiera explicar porque no ha sido recomendada como parte del tratamiento eje de la enfermedad. ${ }^{19}$

El objetivo de la presente investigación fue evaluar si existe alguna asociación entre la cantidad de fibra dietética de la dieta habitual y la actividad actual de la enfermedad en pacientes con $\mathrm{CUCl}$.

\section{Métodos}

Se realizó un estudio transversal, anidado en una cohorte de pacientes con $\mathrm{CUCl}$ atendidos en la Clínica de Enfermedad Inflamatoria Intestinal de una institución de tercer nivel entre enero y marzo de 2020.

Con base en el consumo de fibra promedio en pacientes con $\mathrm{CUCl},{ }^{20}$ el tamaño de la muestra estimado fue de 151 pacientes, quienes debían tener diagnóstico histopatológico de $\mathrm{CUCl}$ en cualquier grado de actividad clínica, con cualquier comorbilidad, mexicanos, mayores de 18 años de edad, de uno $u$ otro sexo y que acudían rutinariamente a vigilancia. Fueron descartados los pacientes que no completaron los cuestionarios, que decidieron no participar, que tuvieron colectomía completa o que se encontraban consumiendo algún tipo de fitoterapia (Figura 1). ${ }^{21}$

Se invitó a los pacientes presencialmente y por llamada telefónica, previo consentimiento 0 asentimiento informado. Se les aplicaron cuestionarios de datos sociodemográficos, de la enfermedad y de registro de la dieta habitual, los cuales fueron llenados por el encuestador para evitar posibles sesgos de respuesta; los datos fueron guardados con un folio asignado a cada individuo para resguardar la confidencialidad de la información. La aplicación de los cuestionarios requirió aproximadamente 15 minutos. Los datos fueron procesados con el paquete estadístico SPSS versión 24.0. Los datos sociodemográficos y de la enfermedad se confirmaron mediante la revisión de los expedientes clínicos electrónicos; el cálculo manual de gramos de fibra fue realizado a partir de datos publicados..$^{22}$ El protocolo fue aprobado por los comités de ética e investigación del Instituto Nacional de Ciencias Médicas y Nutrición "Salvador Zubirán" con registro 3247.

La actividad de la enfermedad fue registrada si por lo menos se reunía un criterio de cuatro: actividad clínica, bioquímica, endoscópica o histológica en un 


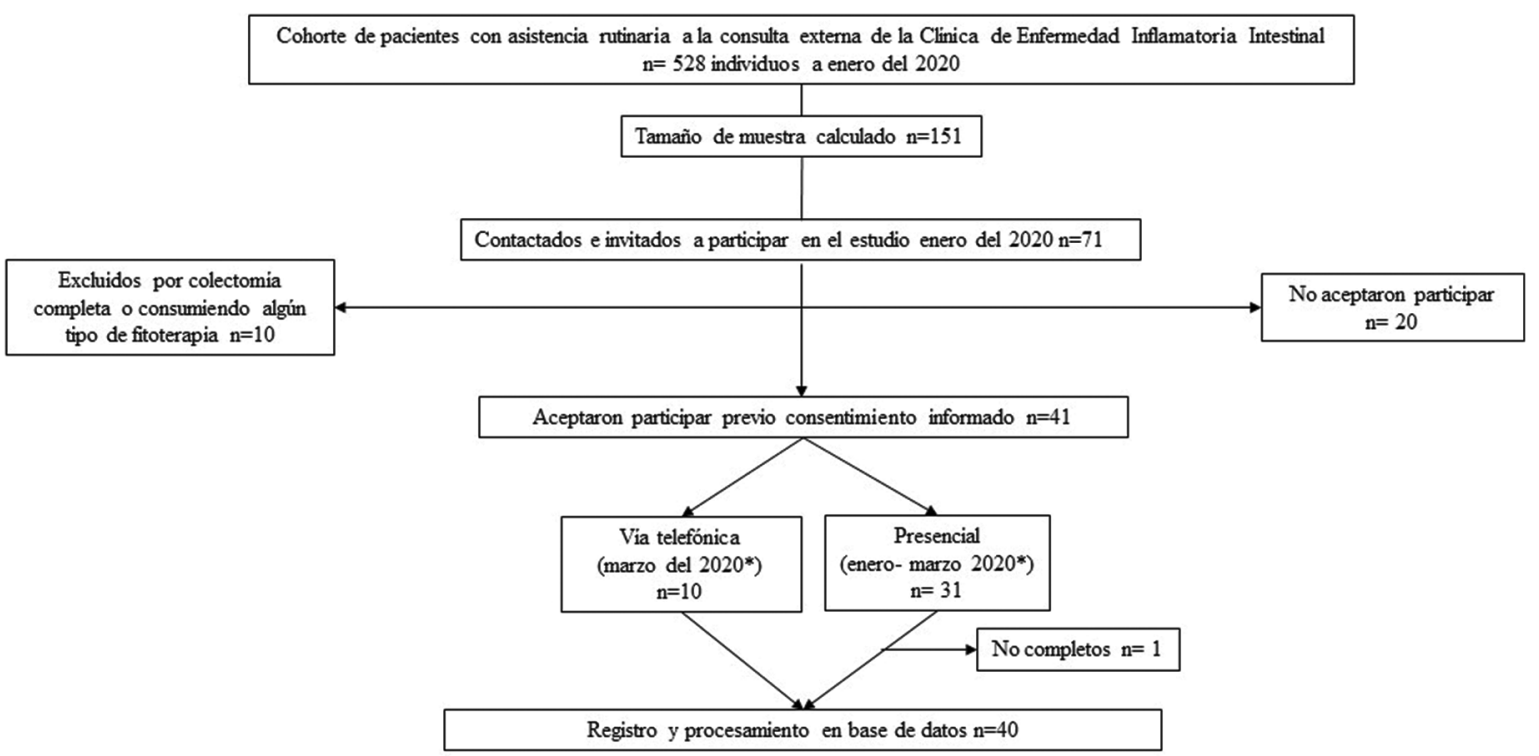

*por confinamiento por Covid19, se declaró oficialmente el 19 de marzo del 2020 en México (21)

Figura 1. Diagrama de flujo de la selección de pacientes con colitis ulcerativa crónica idiopática.

Tabla 1. Características demográficas de la población total estudiada y por grupos, de acuerdo con la actividad actual de la enfermedad

\begin{tabular}{|c|c|c|c|c|c|c|c|}
\hline Variable & \multicolumn{2}{|c|}{$\begin{array}{c}\text { Total } \\
(n=40)\end{array}$} & \multicolumn{2}{|c|}{$\begin{array}{l}\text { Con actividad actual } \\
\qquad(n=24)\end{array}$} & \multicolumn{2}{|c|}{$\begin{array}{l}\text { Sin actividad actual } \\
\qquad(n=17)\end{array}$} & $p^{*}$ \\
\hline \multirow[t]{2}{*}{ Sexo femenino, $n(\%)$} & \multicolumn{2}{|c|}{$25 / 41$} & \multicolumn{2}{|c|}{$14(58.3 \%)$} & \multicolumn{2}{|c|}{$11(64.7 \%)$} & 0.680 \\
\hline & Mediana & RIC & Mediana & RIC & Mediana & RIC & \\
\hline Edad & 40 & $33-53$ & 41 & $33-52$ & 38 & $28-56$ & 0.474 \\
\hline Años de evolución & 12 & $5-15$ & 8.5 & 3-15 & 12 & $7-17$ & 0.100 \\
\hline Número de recaídas & 0 & $0-1$ & 1 & $0-1$ & 0 & $0-0$ & 0.118 \\
\hline Gramos de fibra dietética & 19 & $14-28$ & 16 & $10-34$ & 20 & $18-26$ & 0.062 \\
\hline
\end{tabular}

RIC: rango intercuartílico. *U de Mann-Whitney

periodo menor a tres meses al momento de la entrevista y realización de estudios de laboratorio o colonoscopia con biopsias. En cuanto al registro de alimentos, los pacientes se dividieron para el análisis en dos grupos con base en la recomendación de consumo (14 g/1000 kcal); con la prueba de U MannWhitney se buscaron diferencias entre ellos. Las variables cuantitativas se trataron con mediana y rangos intercuartílicos y las cualitativas, con frecuencias y porcentajes. El análisis de correlación de Spearman permitió identificar el número de recaídas de acuerdo con la cantidad de fibra dietética consumida y con regresión logística fue posible determinar la relación del consumo de fibra dietética con la actividad actual de la enfermedad.

\section{Resultados}

Debido a la pandemia de COVID-19 se lograron incluir 40 pacientes, quienes tuvieron mediana de edad de 40 años y la mayor parte fue del sexo femenino $(n=25)$. Se encontró que en la actividad de la enfermedad no influyeron variables como la edad $(p=0.474)$, el sexo $(p=0.680)$, los años de evolución $(p=0.100)$ ni el número de recaídas $(p=0.118)$. También se encontró que el grupo sin actividad actual de la enfermedad consumió mayor cantidad de fibra dietética: $20 \mathrm{~g}, \mathrm{RIC}=18-26$ (Tabla 1).

Al dividir a los pacientes conforme al consumo de fibra, se observó que quienes cumplieron con la cantidad recomendada presentaron menos actividad 


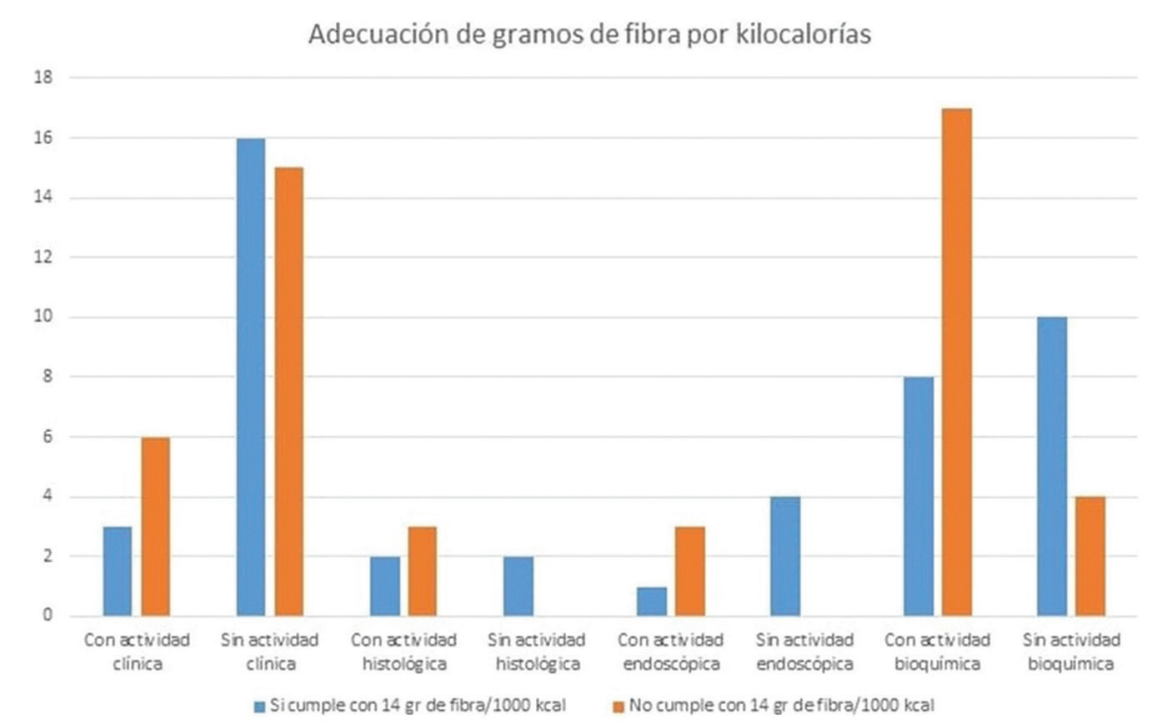

Figura 2. Adecuación por grupos de consumo de fibra con actividad y sin actividad de la colitis ulcerosa crónica idiopática.

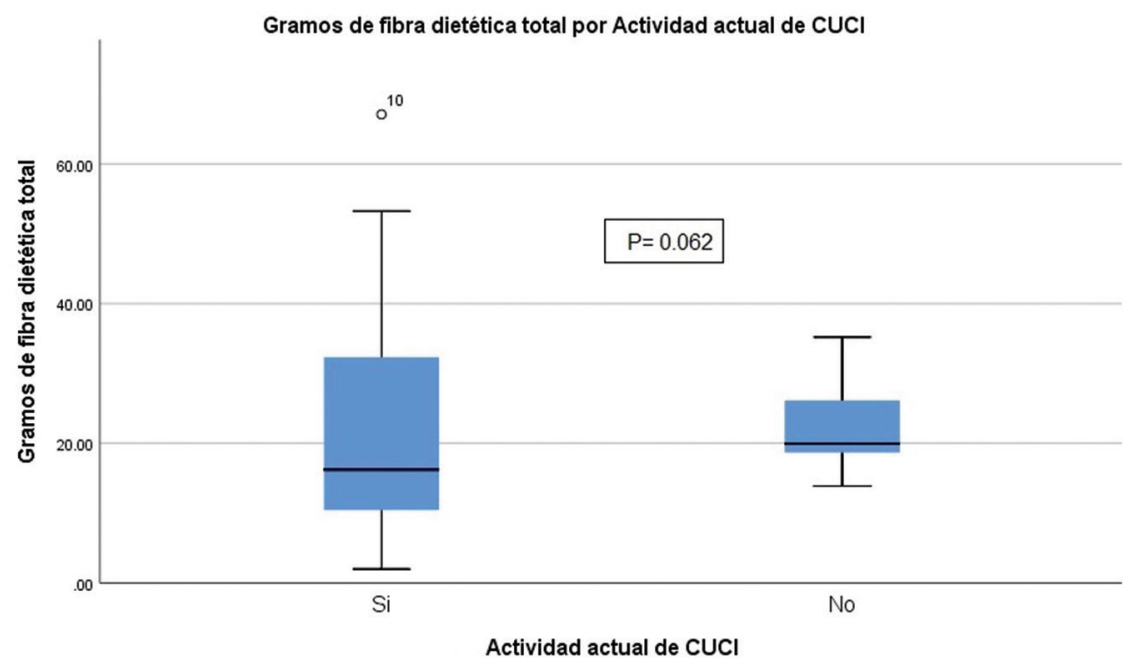

Figura 3. Diferencia entre grupos por consumo de gramos de fibra.

clínica, bioquímica, histológica y endoscópica de la enfermedad (Figura 2). Se pudo establecer que $47 \%$ de los pacientes consumió más de $14 \mathrm{~g} / 1000 \mathrm{kcal} / \mathrm{día}$ y el análisis de regresión logística mostró que dicho consumo se asoció como factor protector hacia la actividad actual de la enfermedad (RM $=0.227$, IC $95 \%=0.059-0.882, p=0.032$ ). En la Figura 2 se puede observar la diferencia entre grupos por gramos de fibra dietética: es evidente que quienes presentaron actividad actual no cumplieron con la recomendación del consumo de fibra $(p=0.062)$. El consumo de fibra tuvo una relación inversamente proporcional a la actividad bioquímica de la enfermedad, como se puede observar en las Figuras 3 y $4(r=-0.379$, $p=0.017)$.

Respecto al efecto de la fibra en el número de recaídas en el último año, también se pudo apreciar una correlación inversamente proporcional entre estas ( $r=-0.399, p=0.011)$, como se muestra en la Figura 5.

\section{Discusión}

Conforme los resultados obtenidos, parece que la fibra tiene un efecto que favorece el mantenimiento de la remisión y que cumplir con la cantidad establecida 


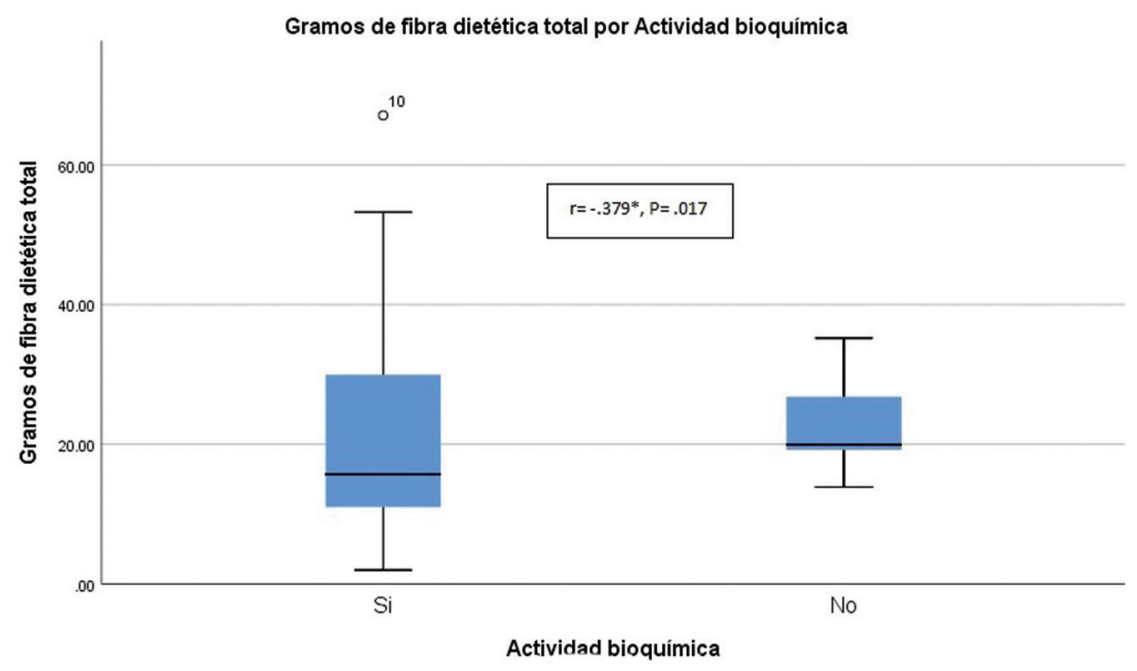

Figura 4. Diferencia por grupos entre actividad bioquímica y gramos de fibra dietética total.

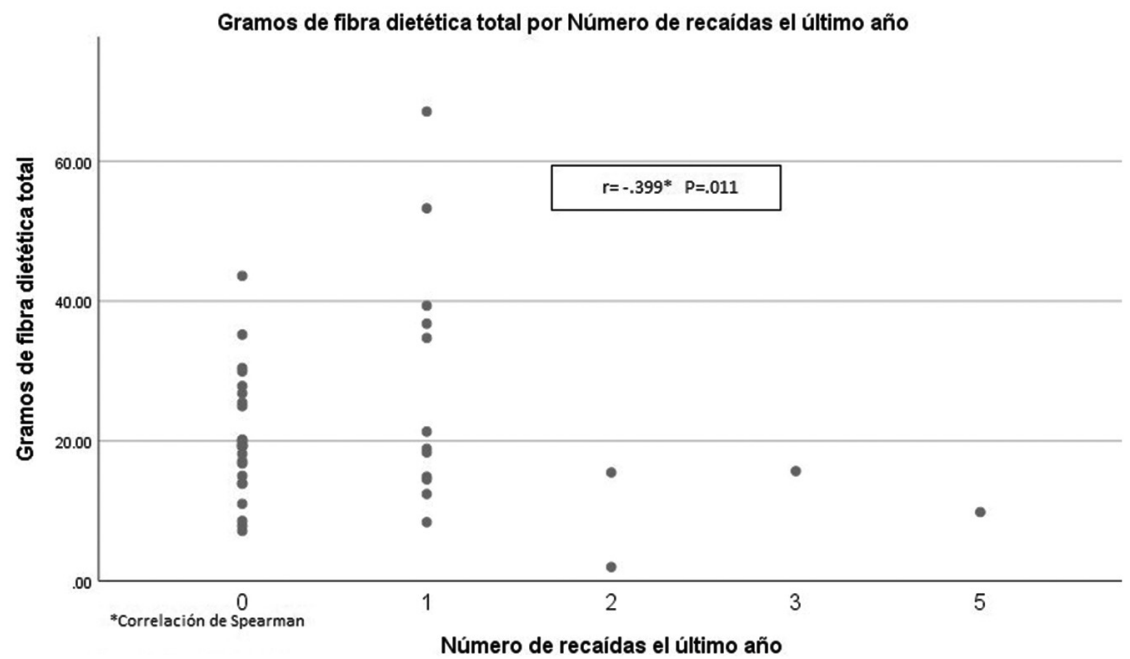

Figura 5. Diagrama de dispersión del número de recaídas por gramos de fibra dietética total.

como meta para este tipo de enfermos no representa una barrera, ya que casi la mitad de la población estudiada pudo alcanzarla.

En un estudio que apoya estos hallazgos,,$^{20}$ en el cual se recopilaron datos de una encuesta dietética completa a 489 participantes con $\mathrm{CUCl}$ o colitis indeterminada en remisión al inicio de la investigación y que se completó seis meses después, se observó que los participantes con mayor duración de la enfermedad, antecedentes de cirugía y hospitalización previa por enfermedad inflamatoria intestinal consumieron menos fibra. Las estimaciones del efecto sugirieron que el alto consumo de fibra no se asoció a la probabilidad de recaída, con RM ajustada para el cuartil 4 (24.5 g/día) versus el cuartil 1 (10.8 g/día) de 1.38 (IC $95 \%=0.74-2.60)$ y 1.82 (IC $95 \%$ 1.05-21.66), respectivamente. Los autores encontraron que el consumo recomendado de fibra fungió como factor protector en la actividad actual de la enfermedad (RM $=0.227$, IC $95 \%=0.059$ $0.882, p=0.032$ ) y que los gramos de fibra dietética consumidos se correlacionaron negativamente con la actividad bioquímica de la enfermedad $(r=.379$, $p=0.011) .{ }^{20}$

En un ensayo clínico aleatorizado, a 102 pacientes con $\mathrm{CUCl}$ en remisión distribuidos aleatoriamente en tres grupos se les proporcionó un suplemento de semillas de Plantago ovata ( $\mathrm{PO}=10 \mathrm{~g}$ dos veces al 
día), mesalazina ( $\mathrm{M}=500 \mathrm{mg}$ tres veces al día) y Plantago ovata + mesalazina $(\mathrm{PO}+\mathrm{M}=$ mismas dosis) para estudiar el papel de la fibra fermentable. El resultado primario de eficacia fue el mantenimiento de la remisión durante 12 meses. Después del periodo, el grupo PO tuvo 60 \% de recaída; el grupo M, 65 \%; y el grupo $\mathrm{PO}+\mathrm{M}, 70 \%$; además, se observó un aumento significativo en los niveles de butirato fecal ( $p=0.018$ ) después de la administración de semillas de $\mathrm{PO}$, por lo que se concluyó que podrían ser tan efectivas como la mesalazina para mantener la remisión. ${ }^{6}$

Con los estudios anteriores confirmamos que los resultados de nuestra investigación van en el camino correcto.

A pesar de los posibles beneficios, la fermentación colónica puede aumentar el dolor abdominal y la flatulencia debido a que ese proceso produce gas, por lo que el consumo de fibra por arriba de la recomendación debe evaluarse si dichas molestias se presentan moderada o intensamente. Es necesario recordar que durante la actividad de la $\mathrm{CUCl}$, el sangrado y, por lo tanto, el oxígeno favorecen el crecimiento de bacterias productoras de ácido láctico, lo que aunado a un pH ácido que no ayuda a la fermentación bacteriana lleva a que la producción de lactato sea mayor que la de butirato. ${ }^{23}$ Por ello, en esa etapa se sugiere no consumir fibra por arriba de la recomendación para la población general.

Entre las limitantes del estudio que dificultaron completar la muestra destacó la pandemia ocasionada por el coronavirus SARS-CoV-2, ya que las actividades del Instituto Nacional de Ciencias Médicas y Nutrición "Salvador Zubirán" fueron canceladas hasta nuevo aviso al convertirse en un centro COVID. Otra limitante fue que no se cuantificó la cantidad de fibra soluble e insoluble por separado; se recomienda que para investigaciones futuras se evalúe y clasifique el tipo de fibra que consumen los pacientes durante ese tiempo y se compare con la dieta de pacientes que estén en algún grado de actividad de la enfermedad, o realizar un estudio de seguimiento con un mayor tamaño de muestra, lo cual sería ideal para conocer el impacto de este nutrimento en diferentes momentos de la CUCI.

\section{Conclusión}

Este estudio piloto permite concluir que el consumo recomendado de fibra en la dieta habitual para la población general tiene un efecto protector contra la actividad actual de la enfermedad en pacientes mexicanos con CUCl.

\section{Agradecimientos}

Los autores agradecen al personal médico, practicantes de nutrición y personal del Departamento de Gastroenterología del Instituto Nacional de Ciencias Médicas y Nutrición "Salvador Zubirán", por el apoyo para que la presente investigación se llevara a cabo.

\section{Financiamiento}

La presente investigación no recibió apoyo específico proveniente de agencias del sector público, sector comercial o entidades sin ánimo de lucro.

\section{Conflicto de intereses}

Los autores no tienen conflicto de intereses.

\section{Responsabilidades éticas}

Protección de personas y animales. Los autores declaran que para esta investigación no se realizaron experimentos en seres humanos ni en animales.

Confidencialidad de los datos. Los autores declaran que siguieron los protocolos de su centro de trabajo sobre la publicación de datos de pacientes.

Derecho a la privacidad y consentimiento informado. Los autores obtuvieron el consentimiento informado de los pacientes o sujetos referidos en el artículo. Este documento obra en poder del autor de correspondencia.

\section{Bibliografía}

1. De León-Rendón J, Jiménez-Bobadilla B, López-Pérez RY, Gracida-Mancilla NI, Alarcón-Bernés L, Villanueva-Herrero JA. Colitis ulcerosa crónica idiopática: epidemiología, características clínicas y factores asociados al tratamiento quirúrgico en un hospital de tercer nivel en México. Cir Cir. 2019;87:450-458.

2. Feldman M, Friedman LS, Brandt LJ. Sleisenger y Fordtran. Enfermedades digestivas y hepáticas. Fisiopatología, diagnóstico y tratamiento. EE. UU.: Elsevier; 2008.

3. Yamamoto-Furusho JK. Clinical epidemiology of ulcerative colitis in Mexico. A single hospital-based study in a 20-year period (1987-2006). J Clin Gastroenterol. 2009;43:221-224.

4. Yamamoto-Furusho JK, Bosques-Padilla F, de-Paula J, Galiano M.T, Ibáñez $P$, Juliao $F$, et al. Diagnóstico y tratamiento de la enfermedad inflamatoria intestinal: Primer Consenso Latinoamericano de la Pan American Crohn's and Colitis Organisation. Rev Gastroenterol Mex. 2016;82:46-84.

5. Vergara-Fernández O, Takahashi-Monroy T, González-Contreras QH. Conceptos actuales en colitis ulcerativa crónica inespecífica. Cir Gen. 2006;28:42-49.

6. Fernández-Bañares $F$, Hinojosa J, Sánchez-Lombraña JL, Navarro E, Martínez-Salmerón JF, García-Pugés A, et al. Randomized clinical trial of Plantago ovata seeds (dietary fiber) as compared with mesalamine in maintaining remission in ulcerative colitis. Spanish Group for the Study of Crohn's Disease and Ulcerative Colitis (GETECCU). Am J Gastroenterol. 1999;94:427-433.

7. García-Peris P. La fibra en la alimentación. Ámbito hospitalario. España: Grafiques Celler; 2004.

8. Kaplan GG. Does consuming the recommend daily level of fiber prevent Crohn's disease? Gastroenterology. 2013;145:925-927. 
9. Bach K, Knud E, Lærke, Helle N, Hedemann, Mette S. Impact of diet-modulated butyrate production on intestinal barrier function and inflammation. Nutrients. 2018;10:1499.

10. Yates CM, Calder PC, Rainger GE. Pharmacology and therapeutics of omega-3 polyunsaturated fatty acids in chronic inflammatory disease. Pharmacol Ther. 2014;141:272-282.

11. Bischoff S, Escher J, Hebuterne X, Kłe $\mathrm{c}$ S, Krznaric Z, Schneider S et al. ESPEN practical guideline: Clinical Nutrition in inflammatory bowel disease. Clin Nutr. 2020;39:632-653.

12. Sevilla L. Influencia de la alimentación en la enfermedad inflamatoria intestinal. Cataluña: Universitat Oberta de Catalunya; 2018.

13. Gentschew L, Ferguson LR. Role of nutrition and microbiota in susceptibility to inflammatory bowel diseases. Mol Nutr Food Res. 2012 56:524-535.

14. Roediger WE. The colonic epithelium in ulcerative colitis: an energy-deficient disease? Lancet. 1980;2:712-715.

15. Kim Y. Short-chain fatty acids in ulcerative colitis. Nutrition Rev. 1998:56:17-24

16. Inan MS, Rasoulpour EJ, Yin L, Hubbard AK, Rosenberg DW, Giardina C. The luminal short-chain fatty acid butyrate modulates NF-kB activity in a human colonic epithelial cell line. Gastroenterology. 2000; 118:724-734.
17. Segain JP, Raingeard de la Blétière D, Bourreille A, Leray V, Gervois N, Rosales $C$, Ferrier $L$, et al. Butyrate inhibits inflammatory responses through NFkB inhibition: implications for Crohn's disease. Gut. 2000:47:397-403.

18. Sánchez R, Martín M, Palma S, López B, Bermejo LM, Gómez C. Indicaciones de diferentes tipos de fibra en distintas patologías. Nutr Hosp. 2015;31:2372-2383.

19. Bernstein CN, Eliakim A, Fedail S, Fried M, Gearry R, Goh KL, et al. World Gastroenterology Organisation Global Guidelines Inflammatory Bowel Disease: update August 2015. J Clin Gastroenterol. 2016;50:803-818.

20. Brotherton CS, Martin CA, Long MD, Kappelman MD, Sandler RS. Avoidance of fiber is associated with greater risk of Crohn's disease flare in a 6-month period. Clin Gastroenterol Hepatol. 2016:14:1130-1136.

21. Acuerdo por el que el Consejo de Salubridad General reconoce la epidemia de enfermedad por el virus SARS- Cov2 (Covid19) en México, como una enfermedad grave de atención prioritaria, así como se establecen las actividades de preparación y respuesta ante dicha epidemia [Internet]. México: Diario Oficial de la Federeción; 2021.

22. Morales-De León J, Bourges-Rodríguez H, Camacho-Parra ME. Tablas de composición de alimentos y productos alimenticios. México: Instituto Nacional de Ciencias Médicas y Nutrición Salvador Zubirán; 2015

23. Vernia P, Caprilli R, Latella G, Barbetti F, Magliocca FM, Cittadini M. Fecal lactate and ulcerative colitis. Gastroenterology. 1988;95:1564-1568. 